\title{
EXPERIMENTAL APPROACH TO THE DESIGN OF THE LINKS OF A DELTA ROBOT
}

\author{
RICARDO CASTILLO, OSCAR F. AVILES \& MAURICIO F. MAULEDOUX
}

Research Scholar, DAVINCI Research Group, Department of Mechatronics Engineering, Universidad Militar

Nueva Granada, Bogota, Colombia

\begin{abstract}
This paper shows the mathematical development of a Delta parallel robot of four Degrees Of Freedom (DOF) to obtain the length of its links, solving its direct and inverse kinematics and the Jacobian matrix. Also, an algorithm to find the workspace's robot is posed and finally, the dimension links are obtained by six different kinds of multi-objective genetic algorithm to maximize the workspace and dexterity of the robot.

KEYWORDS: Delta Parallel Robot, Direct and Inverse Kinematic, Jacobian Matrix, Multi-Objective Genetic Algorithm, Workspace \& Dexterity
\end{abstract}

Received: Feb 25, 2020; Accepted: Mar 17, 2019; Published: May 04, 2020; Paper Id.: IJMPERDJUN202030

\section{INTRODUCTION}

A robot is designed to be controlled by a computer or similar device to be able to perform multiple tasks by simply changing its programming [1]. In the beginning, the robots performed simple and monotonous tasks in work environments dangerous to man, achieving a reduction in the costs and manufacturing times of products and increasing their quality and productivity [2]. Currently, robots are essential in industrial processes, being useful in operations such as welding, painting, machining operations (drilling, milling, grooving, etc.), grinding, polishing, brushing, cutting, product quality control, among others [3].

Until a few years ago, open-chain manipulators were responsible for performing the tasks named above. A serial manipulator is formed from a single kinematic chain and its mechanical structure resembles that of the human arm (Figure 1). In these robots, the actuators are located along with its mechanical structure which generates an increase in the inertia that the robot must overcome to move, that is, the more actuators the robot has, the greater the load on it, affecting the stability of the whole mechanism. In addition to this, each actuator increases the probability of generating a position and orientation error of the final effector [4].

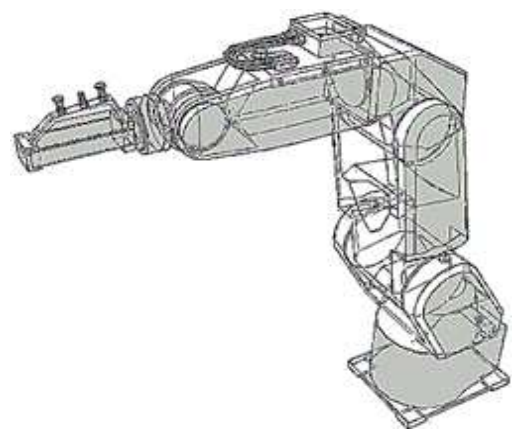

Figure 1: Serial Manipulator. 
Parallel manipulators emerged (Figure 2), where two or more kinematic chains support the mobile platform increasing rigidity, stability, load capacity, the accuracy of position and orientation of the final effector, speed and the acceleration of robot movements [5]. These advantages make these robots suitable for applications such as:

- $\quad$ Pick and place for packaging, packaging or quality control operations in the food industry.

- $\quad$ Micro movement robots.

- Motion simulators.

- Haptic interfaces.

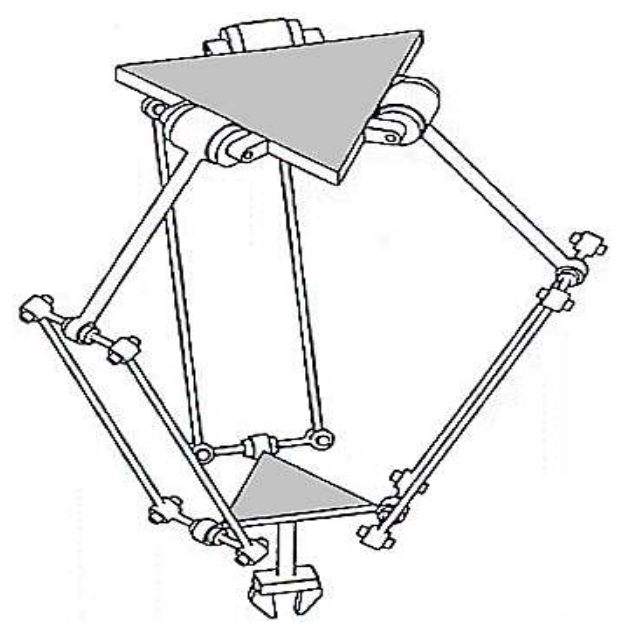

Figure 2: Parallel Manipulator.

However, compared to serial manipulators, parallel manipulators have three disadvantages: difficulty in the development of their kinematics, less workspace, and increased singularities, which hinders the performance of the robot in its environment [5]. Over the years, different solutions have been proposed to the development of the kinematics of parallel robots such as the Delta type (Figure 2). One way to solve the kinematics is through an analysis of its geometry [6], [7] or using software such as ADAMS [8].

To solve the problem of reduced workspace, new forms of parallel robots have been developed, where revolution joints are combined with binoculars [9] or the prismatic joints replace those of revolution [10], [11]. These new configurations achieve a greater workspace, but the robot's movement speed is reduced because the linear actuators cannot reach the speeds that the revolution actuators reach. Algorithms have also been implemented to maximize the workload of parallel robots using genetic algorithms [5], [9], [12], [13], the SQP method [14], the CRS method [15], between others.

The objective of this work is to show how the workspace of a 4 DOF Delta robot can be optimized using multiobjective genetic algorithms. Section 2 presents the solution of direct kinematics, inverse kinematics and the Jacobian matrix of the robot. Section 3 presents an algorithm to find the robot's workspace. In section 4, you will find the dimensions of the robot links to optimize your workspace. Finally, section 5 presents the conclusions.

\section{DELTA ROBOT KINEMATICS}

To obtain a mathematical model that describes the kinematics of the robot, its geometry must first be analyzed. As seen in Figure 3, the Delta robot consists of a fixed base, three servomotors $\left(R_{1}, R_{2} \mathrm{y} R_{3}\right)$ and at the same distance from the center 
of the base and separated $120^{\circ}$ each other, a mobile base and three kinematic chains, each consisting of two links, which are connected employing universal joints $(\mathrm{U})$. The reference system $X Y Z$ is located in the center of the fixed base, with the axis $X$ perpendicular to the axis of the servomotor $R_{1}$ and the $Z$ axis is perpendicular to the base.

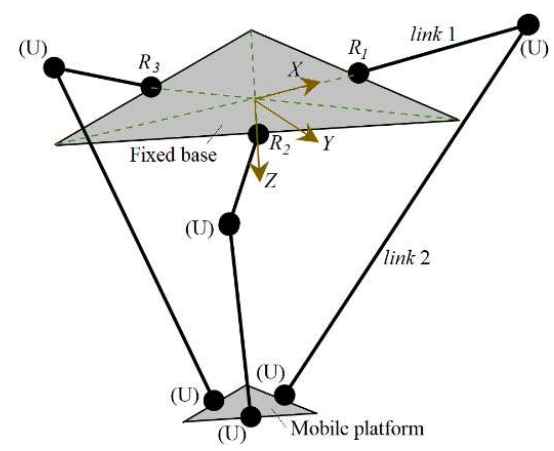

Figure 3: Delta Robot Structure.

In Figure 4, it can be seen that the link $L_{1}$ is attached to the fixed base through the joint $A_{i}$ and to the link $L_{2}$ by the joint $B_{i}$. The link $L_{2}$ joins the mobile platform through articulation $C_{i}$. Finally, $B_{i}$ is the angle between link $L_{1}$ and $L_{2}$. The center of the mobile base represents the position of the Delta robot $P=\left[P_{x} P_{y} P_{z}\right]^{T}$.

The distance between $O$ and $A_{1}, A_{2}$ and $A_{3}$ is $R$ and the distance between $P$ and $R_{1}, R_{2}$ or $R_{3}$ is $r$

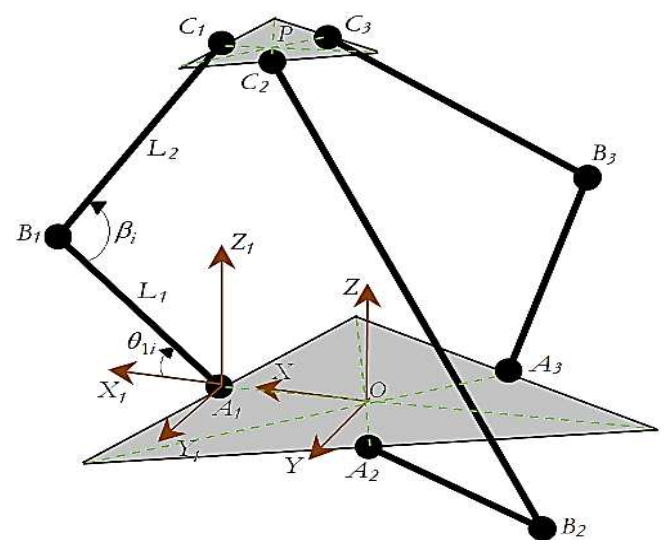

Figure 4: Structure of the Delta Robot.

Below are the vectors that will be used to calculate the kinematics of the robot $^{1}$ :

$$
\overrightarrow{O A_{1}}=\left[\begin{array}{c}
R c \phi_{i} \\
R c \phi_{i} \\
0 \\
1
\end{array}\right]
$$

\section{Inverse Kinematics}

Inverse kinematics allows knowing the variables of the actuators if the position of the mobile base is known. Thanks to the symmetry of the robot, each kinematic chain can be analyzed independently. As can be seen in Figure 5 there are in total there are nine joint angles: $\theta_{1 i}, \theta_{2 i}, \theta_{3 i}$ for $i=1,2,3$ associated to the manipulator, being $\theta_{11}, \theta_{12}, \theta_{13}$ the variables of the actuator joints.

$\operatorname{sen} \theta=\mathrm{s} \theta$, and $\cos \theta=\mathrm{c} \theta$ 
${ }^{1}$ Notation. $\operatorname{sen} \theta=\mathrm{s} \theta$, and $\cos \theta=\mathrm{c} \theta$

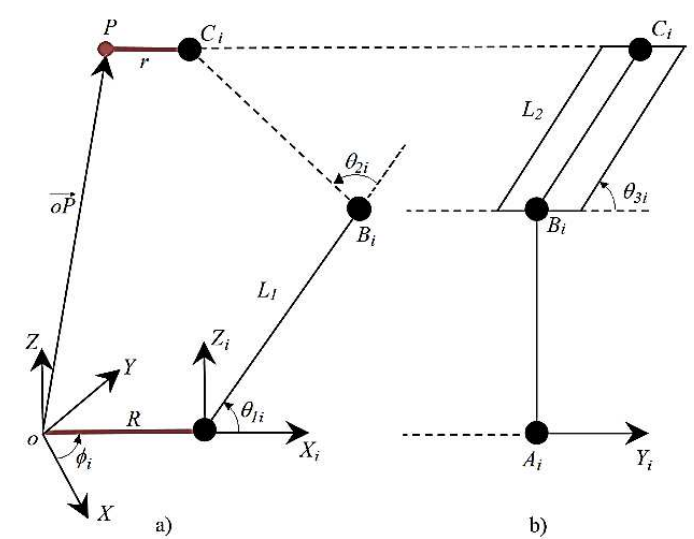

Figure 5: Description of the Angles: a) Front View and) Lateral View.

It is possible to form a triangle with vertices $A_{i} B_{i} C_{i}$ as shown in Figure 6 and the magnitude $A_{i} C_{i}$ can be found by means of the cosine theorem:

$$
A_{i} C_{i}^{2}=L_{1}^{2}+L_{2}^{2}-2 L_{1} L_{2} \cos \beta_{i}
$$

$A_{i} C_{i}^{2}=L_{1}^{2}+L_{2}^{2}-2 L_{1} L_{i}$

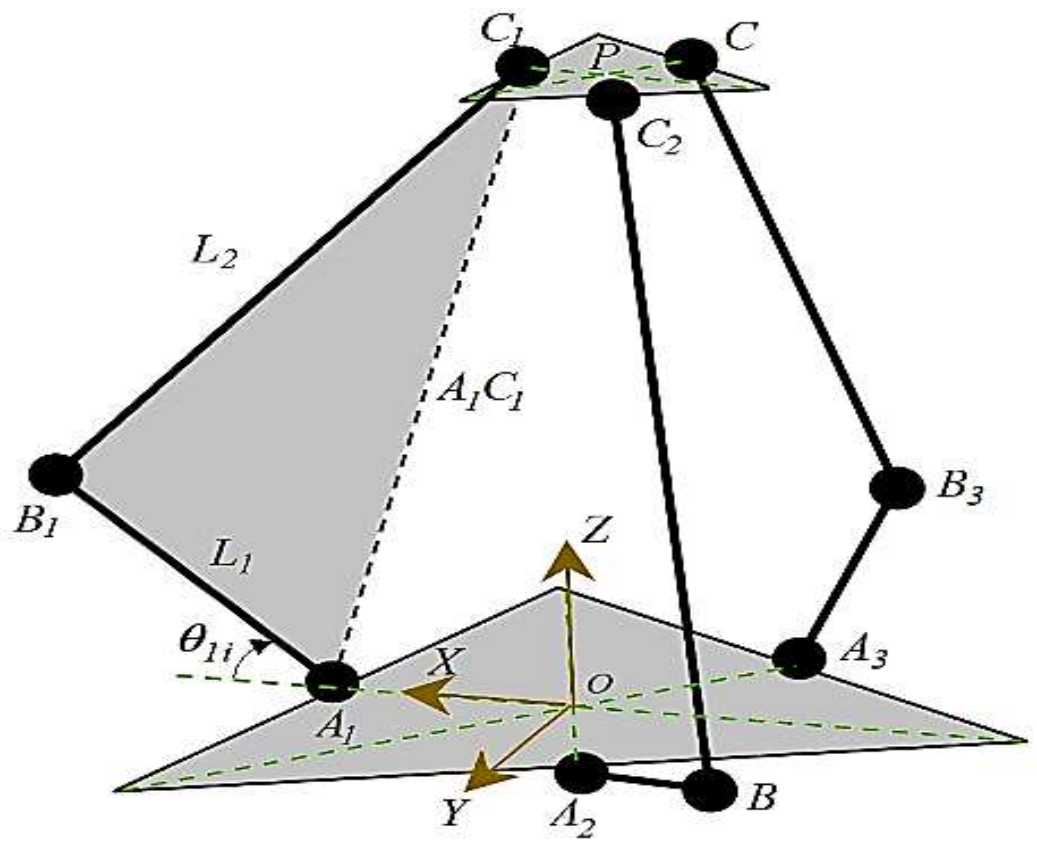

Figure 6: Vector Representation of the First Kinematic Chain.

Applying the definition of the scalar product is found $\mathrm{c} \beta_{i}$ :

$$
\mathrm{c} \beta_{i}=\frac{\overrightarrow{B_{l} A_{l}} \cdot \overrightarrow{B_{l} C_{l}}}{L_{1} L_{2}}
$$

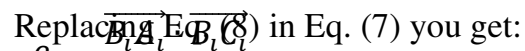

$\mathrm{c} \beta_{i}=\frac{L_{1} L_{2}}{A_{i} C_{i}^{2}=L_{2}^{2}-L_{1}^{2}+2 L_{1} \mathrm{c} \theta_{1 i}\left(P_{X} \mathrm{c} \phi_{i}+P_{Y} \mathrm{~s} \phi_{i}+r-R\right)+2 L_{1} \mathrm{~s} \theta_{1 i} P_{Z}}$

The magnitude of the vector Eq. (6) is: 


$$
A_{i} C_{i}^{2}=\left(P_{X}+\mathrm{c} \phi_{i}(r-R)\right)^{2}+\left(P_{Y}+\mathrm{s} \phi_{i}(r-R)\right)^{2}+P_{Z}^{2}
$$

Equating Eq. (9) and Eq. (10) you get an equation of the form:

$$
a_{i} \mathrm{~s} \theta_{1 i}+b_{i} \mathrm{c} \theta_{1 i}=c_{i}
$$

Where,

$c a_{i}=2 P_{Z} L_{1}$

$c b_{i}=2 L_{1}\left(P_{X} c \phi_{i}+P_{Y} s \phi_{i}+r-R\right)$

$c c_{i}=\left(P_{X}+c \phi_{i}(r-R)\right)^{2}+\left(P_{Y}+s \phi_{i}(r-R)\right)^{2}+P_{Z}^{2}+L_{1}^{2}-L_{2}^{2}$

It is possible to rewrite Eq. (11) taking into account the trigonometric identity of sum of sines and cosines:

$$
\theta_{1 i}=\operatorname{sen}^{-1}\left(\frac{c c_{i}}{\sqrt{c a_{i}^{2}+c b_{i}^{2}}}\right)-\tan ^{-1}\left(\frac{c b_{i}}{c a_{i}}\right)
$$

$\theta_{1 i}=\operatorname{sen}^{-1}\left(\frac{\text { th }}{\left(\text { is wagathe values of } \theta_{11}\right.}\left(\frac{\theta b_{t}}{\sqrt{c a_{i}^{2}+c b_{i}^{2}}}\right)-\tan ^{-1} \theta_{13}\right.$ if the position of the mobile base is known.
Direct Kinematics

The problem of direct kinematics consists in determining the position and orientation of the final effector of the robot from a reference coordinate system. The magnitude of the vector Eq. (5) is $L_{2}$ :

$$
B C_{i}=\left(P_{X}-\mathrm{c} \phi_{i}\left(R+L_{1} \mathrm{c} \theta_{1 i}-r\right)\right)^{2}+\left(P_{Y}-\mathrm{s} \phi_{i}\left(R+L_{1} \mathrm{c} \theta_{1 i}-r\right)\right)^{2}+\left(P_{z}-L_{1} \mathrm{~s} \theta_{1 i}\right)^{2}=L_{2}^{2}
$$

Eq. (13) represents three nonlinear equations that, when solved, allow us to find the position of the mobile base if the angles are known $\theta_{11}, \theta_{12}, \theta_{13}$.

$$
P_{Y}=\frac{A+B P_{Z}}{C}
$$

$\stackrel{\text { Where }}{P}_{Y}=\frac{B P_{Z}}{C}$

$A=\frac{a_{3}-b_{3}}{2 a_{1}-b_{1}}-\frac{a_{3}-c_{3}}{2 a_{1}-c_{1}}$

$B=\frac{2\left(c_{2}-a_{2}\right)}{2 a_{1}-c_{1}}-\frac{2\left(b_{2}-a_{2}\right)}{2 a_{1}-b_{1}}$

$C=\frac{\sqrt{3} b_{1}}{2 a_{1}-b_{1}}+\frac{\sqrt{3} c_{1}}{2 a_{1}-c_{1}}$

$P_{X}=D-E P_{Z}$

And

$D=\frac{a_{3}-b_{3}}{2 a_{1}-b_{1}}-\frac{\sqrt{3} b_{1} A}{C\left(2 a_{1}-c_{1}\right)}$

$E=\frac{2\left(b_{2}-a_{2}\right)}{2 a_{1}-b_{1}}+\frac{\sqrt{3} b_{1} B}{C\left(2 a_{1}-b_{1}\right)}$ 
$P_{Z}=\frac{-b+\sqrt{b^{2}-4 a c}}{2 a}$

$\stackrel{\text { and }}{D}=\frac{a_{3}-b_{3}}{2 a_{1}-b_{1}}-\frac{\sqrt{3} b_{1} A}{C\left(2 a_{1}-c_{1}\right)} E=\frac{2\left(b_{2}-a_{2}\right)}{2 a_{1}-b_{1}}+\frac{\sqrt{3} b_{1} B}{C\left(2 a_{1}-b_{1}\right)} P_{Z}=\frac{-b+\sqrt{b^{2}-4 a c}}{2 a}$

$a=E^{2}+\left(\frac{B}{C}\right)^{2}+1$

$b=2\left(\frac{A B}{C^{2}}-D E-a_{1} E-a_{2}\right)$

$c=D^{2}+\left(\frac{A}{C}\right)^{2}+2 a_{1} D-a_{3}$

Additionally,

$$
\begin{array}{llr}
a_{1}=r-R-L_{1} \mathrm{c} \theta_{11} & a_{2}=L_{1} \mathrm{~s} \theta_{11} & a_{3}=L_{2}^{2}-a_{1}^{2}-a_{2}^{2} \\
b_{1}=R-r+L_{1} \mathrm{c} \theta_{12} & b_{2}=L_{1} \mathrm{~s} \theta_{12} & b_{3}=L_{2}^{2}-b_{1}^{2}-b_{2}^{2} \\
c_{1}=R-r+L_{1} \mathrm{c} \theta_{13} & c_{2}=L_{1} \mathrm{~s} \theta_{13} & c_{3}=L_{2}^{2}-c_{1}^{2}-c_{2}^{2}
\end{array}
$$

\section{Jacobian Matrix}

A closed loop equation of the $i_{t h}$ kinematic chain can be: $\overrightarrow{O P}+\overrightarrow{P C_{l}}=\overrightarrow{O A_{l}}+\overrightarrow{A_{l} B_{l}}+\overrightarrow{B_{l} C_{l}}$. If you derive this expression with respect to time and the result is expressed in the system $X_{i} Y_{i} Z_{i}$ is obtained:

$$
\overrightarrow{V_{p}}=\overrightarrow{\omega_{1 \imath}} \times \overrightarrow{A_{l} B_{l}}+\overrightarrow{\omega_{2 l}} \times \overrightarrow{B_{l} C_{l}}
$$

In Eq. (17) $\overrightarrow{\omega_{2 \imath}}$ represents the velocities of the passive joints, therefore, they are eliminated by multiplying both sides of the equation by $\overrightarrow{B_{l} C_{l}}$ what results:

$$
\overrightarrow{V_{p}} \cdot \overrightarrow{B_{l} C_{l}}=\overrightarrow{B_{l} C_{l}}\left(\overrightarrow{\omega_{1 \imath}} \times \overrightarrow{A_{l} B_{l}}\right)
$$

where:

$$
\begin{aligned}
& \overrightarrow{B_{\imath} C_{\imath}}=\left[\begin{array}{c}
L_{2} \mathrm{c} \theta_{3 i} \mathrm{c}\left(\theta_{1 i}+\theta_{2 i}\right) \\
L_{2} \mathrm{~s} \theta_{3 i} \\
L_{2} \mathrm{c} \theta_{3 i} \mathrm{~s}\left(\theta_{1 i}+\theta_{2 i}\right)
\end{array}\right] \\
& \overrightarrow{V_{p}}=\left[\begin{array}{c}
V_{p X} \mathrm{c} \phi_{i}+V_{p Y} \mathrm{~s} \phi_{i} \\
V_{p Y} \mathrm{c} \phi_{i}-V_{p X} s \phi_{i} \\
V_{p Z}
\end{array}\right] \\
& \overrightarrow{\omega_{1 \imath}}=\left[\begin{array}{c}
0 \\
-\dot{\theta}_{i 1} \\
0
\end{array}\right] \\
& \overrightarrow{A_{l} B}=\left[\begin{array}{c}
L_{1} \mathrm{c} \theta_{1 i} \\
0 \\
L_{1} \mathrm{~s} \theta_{1 i}
\end{array}\right]
\end{aligned}
$$

Solving Eq. (18) and simplifying you get:

$$
j_{x i 1} V_{p X}+j_{x i 2} V_{p Y}+j_{x i 3} V_{p Z}=j_{\theta i} \dot{\theta}_{1 i}
$$


where:

$j_{x i 1}=\mathrm{c} \theta_{3 i} \mathrm{c}\left(\theta_{1 i}+\theta_{2 i}\right) \mathrm{c} \phi_{i}-\mathrm{s} \theta_{3 i} \mathrm{~s} \phi_{i}$

$j_{x i 2}=\mathrm{c} \theta_{3 i} \mathrm{c}\left(\theta_{1 i}+\theta_{2 i}\right) \mathrm{s} \phi_{i}-\mathrm{s} \theta_{3 i} \mathrm{c} \phi_{i}$

$j_{x i 3}=\mathrm{c} \theta_{3 i} \mathrm{~s}\left(\theta_{1 i}+\theta_{2 i}\right)$

$j_{\theta i}=L_{1} \mathrm{~s} \theta_{2 i} \mathrm{c} \theta_{3 i}$

The expression obtained in Eq. (19) represents a system of three equations that can be written in the form:

$$
\left[\begin{array}{lll}
j_{x 11} & j_{x 12} & j_{x 13} \\
j_{x 21} & j_{x 22} & j_{x 23} \\
j_{x 31} & j_{x 32} & j_{x 33}
\end{array}\right]\left[\begin{array}{c}
V_{p X} \\
V_{p Y} \\
V_{p Z}
\end{array}\right]=\left[\begin{array}{ccc}
j_{\theta 1} & 0 & 0 \\
0 & j_{\theta 2} & 0 \\
0 & 0 & j_{\theta 3}
\end{array}\right]\left[\begin{array}{l}
\dot{\theta}_{11} \\
\dot{\theta}_{12} \\
\dot{\theta}_{13}
\end{array}\right]
$$

So, the Jacobian matrix of the Delta robot is:

$$
J=\left(J_{\theta}\right)^{-1} J_{x}=\left[\begin{array}{ccc}
j_{\theta 1} & 0 & 0 \\
0 & j_{\theta 2} & 0 \\
0 & 0 & j_{\theta 3}
\end{array}\right]^{-1}\left[\begin{array}{lll}
j_{x 11} & j_{x 12} & j_{x 13} \\
j_{x 21} & j_{x 22} & j_{x 23} \\
j_{x 31} & j_{x 32} & j_{x 33}
\end{array}\right]
$$

It is said that there is a singularity of inverse kinematics when the determinant $J_{\theta}$ is equal to zero; it is said that there is a singularity of inverse kinematics when the determinant of $J_{x}$ is equal to zero; the singularities are said to be combined when the determinants of $J_{\theta}$ and $J_{x}$ are zero.

\section{Robot Dexterity}

The Jacobian matrix allows identifying unwanted configurations in a Delta robot (singularities). Additionally, it allows knowing the skill level of each robot configuration through the condition number of the Jacobian matrix:

$$
\lambda=\|J\| \cdot\left\|J^{-1}\right\|
$$

The local skill of a parallel robot is defined as the inverse of the condition number of the Jacobian matrix [16]:

$$
\varsigma=\frac{1}{\lambda}
$$

$\varsigma=\frac{1}{\lambda} \quad$ If the value of $\varsigma$ is zero it is because the robot is in a unique configuration and if the value of $\varsigma$ is one, the robot configuration is known as isotropic [17]. Given that the $\varsigma$ index allows analyzing only each configuration of the parallel robot, Gosselin, and Angeles in 1991, they proposed an index that allows measuring the dexterity of a robot in its entire workspace (global index):

$$
\eta=\frac{\int_{W} \varsigma d w}{\int_{W} d w}
$$

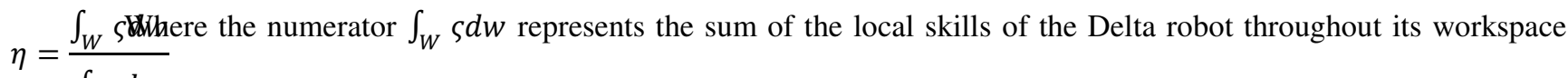
and therdellominator $\int_{W} d w$ is the value of the robot's workspace.

\section{DELTA ROBOT WORKSPACE}

The workspace of a robot is considered as the region in the three-dimensional Cartesian space that can be reached by a point of its final effector [12], in the case of a Delta robot, it is the region in the three-dimensional space that can reach one 
point of your mobile base. Designing a Delta robot for maximum workspace does not guarantee that the robot will be optimal for practical applications and it is feasible that it becomes a manipulator with unwanted kinematic characteristics such as low dexterity and maneuverability [18]. For this reason, in this article, an algorithm was used to calculate the exact amount of points in the space that is capable of reaching the robot's mobile base. The algorithm is based on the solution of direct kinematics for all possible combinations of the actuators, obtaining in each case a point in the space, which is evaluated to know whether it belongs to the robot's workspace. The algorithm also allows you to find the overall skill of the robot's workspace.

First, the local dexterity index of each point that belongs to your workspace is calculated, then all the local indexes are added and divided by the number of points that the final effector of the robot can reach in the space.

\section{OPTIMIZATION OF THE WORKSPACE}

It seeks to find a set of decision vectors; whose elements are the dimensions of the links and that maximize the workspace and skill of the Delta robot. In this way, the objective functions are the number of points that make up the robot's workspace and the global skill index raised by Gosselin and Angeles.

The dimensions of the links must meet the following restrictions:

$$
\begin{gathered}
L_{1}<L_{2} \\
100<L_{1}<350 \\
360<L_{2}<600 \\
L_{1}+L_{2}<900
\end{gathered}
$$

Figure 7 shows the problem-solving space where the blue dots represent the possible dimensions of the links and the red dots are dimensions that do not meet the restrictions outlined above. To find the set consisting of the best solutions, six different methods based on genetic algorithms were proposed:

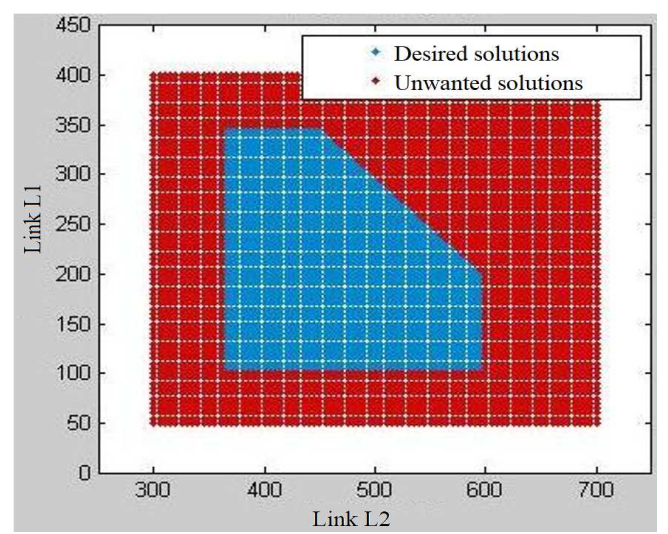

Figure 7: Problem Solving Space.

To find the set consisting of the best solutions, six different methods based on genetic algorithms were proposed:

- $\quad$ RWGA: Random Weight Genetic Algorithm.

- FWGA: Fixed Weight Genetic Algorithm.

- AWGA: Adaptive Weight Genetic Algorithm.

- NSGA: Non-dominated Sorting Genetic Algorithm. 
- $\quad$ NPGA: Niched Pareto Genetic Algorithm.

- MOFGA: Multiplied Objectives Genetic Algorithm.

The first five algorithms are known in the view that many investigations have already been developed around them. The last algorithm is a method proposed in this article where the adaptability value of each individual is found by multiplying the two values of the objective functions to preserve the individuals with the highest amount of points they can reach and the overall skill index higher. Thanks to the fact that the six algorithms are based on genetic algorithms, the following nine steps can be followed to find the solution set of the problem:

Step 1: Generate an initial population of 50 individuals, verifying that the individuals comply with the restrictions of the links $L_{1}$ y $L_{2}$.

Step 2: Evaluate each individual in the two objective functions, that is, obtain the overall skill index and the number of points that make up the Delta robot's workspace for each solution.

Step 3: A tentative set of optimal Pareto solutions is formed, where half corresponds to the ten individuals of the current population with the most points in the space that can reach the mobile base and the other half are the ten individuals with the best overall skill index.

Step 4: An adaptive value is assigned to each individual.

Step 5: Through the selection operator, some individuals are chosen as parents. The greater the adaptability of an individual, the greater the probability of being chosen as a parent. To have more solution options, three selection methods (truncation, stochastic universal sampling, and roulette) were used for each algorithm.

Step 6: $N$ new individuals (offspring) are generated through the intermediate crossing operator.

Step 7: Through the mutation operator, some individuals are chosen randomly to generate small changes.

Step 8: Some individuals are randomly selected from the population to be changed by individuals from the Pareto tentative set.

Step 9: Steps 2 through 9 are repeated until a total of 100 generations are completed.

Once the 18 algorithms were executed, six multi-objective genetic algorithms each for three selection methods, the solution sets were grouped according to the selection method, see Figure 8, Figure 9 and Figure 10.

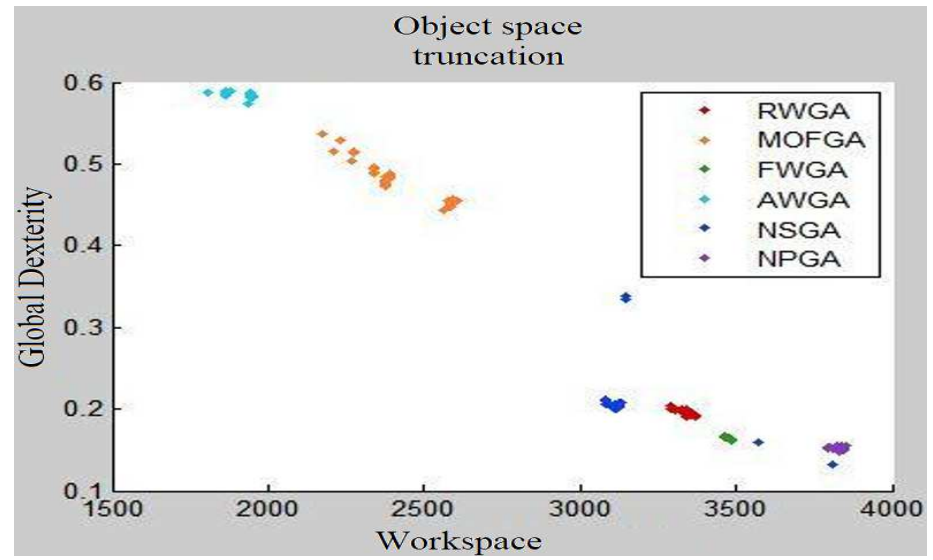

Figure 8: Set of Solutions with the Truncation Method. 


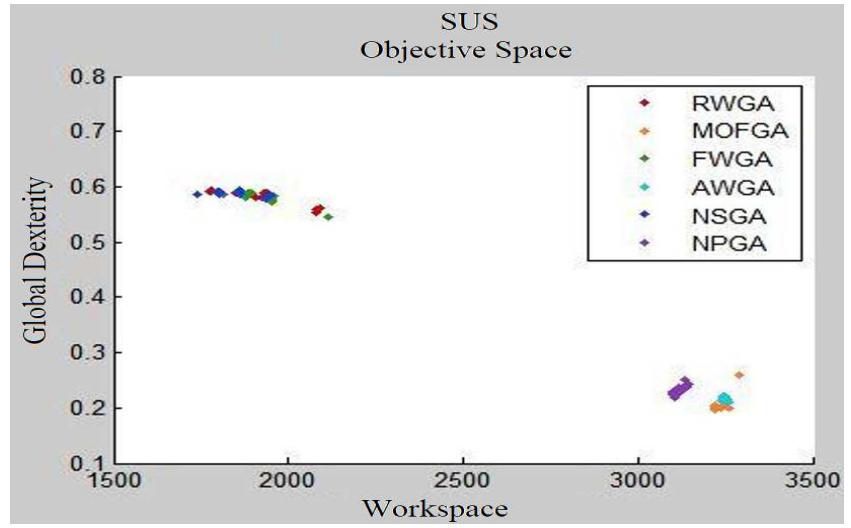

Figure 9: Set of Solutions with the SUS Method.

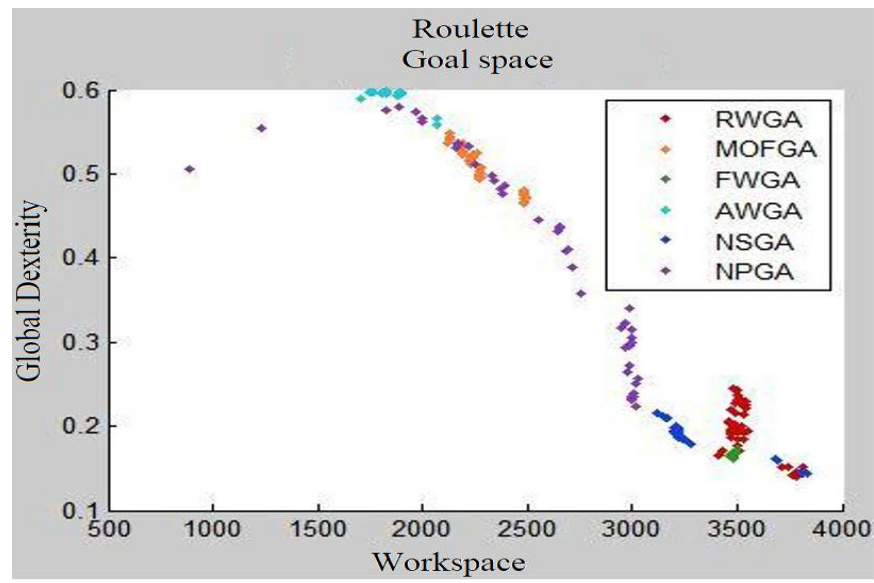

Figure 10: Set of Solutions with the Roulette Method.

To find the final set of solutions, these three sets were united into one and its optimal Pareto boundary (nondominated solutions) was identified, as shown in Figure 11.

Of that Pareto optimal border, individuals with the highest overall skill (greater than 0.47) and the highest number of points in the workspace (more than 200,000 points) are those of interest to obtain the dimensions of the links of the Delta robot Analyzing each of the individuals and their new values of objective functions, the dimensions of the robot links are: $L_{1}=260 \mathrm{~mm}$ y $L_{2}=420 \mathrm{~mm}$.

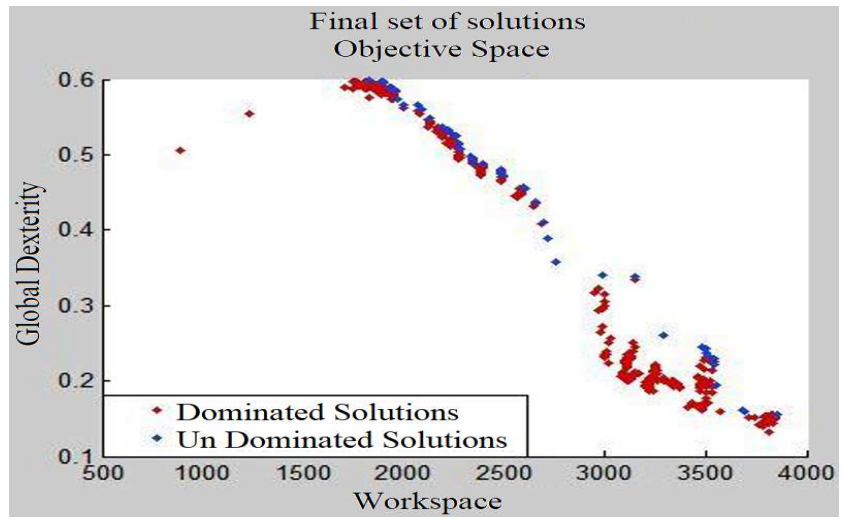

Figure 11: Pareto Front of the Final Set of Solutions, MATLAB. 
Figures 12 to 15 show the different views of the real workspace of the Delta robot, with the dimensions of the links found. The data of this workspace are:

- $\quad$ Points in space: 259656

- $\quad$ Optimum points: 146273

- Global skill: 0,556

- Minimum reach: $X=-342,8015 \mathrm{~mm}, Y=-296,9611 \mathrm{~mm}$ y $Z=105,7695 \mathrm{~mm}$

- $\quad$ Maximum reach: $X=342,4004 \mathrm{~mm}, Y=296,9611 \mathrm{~mm}$ y $Z=677,0132 \mathrm{~mm}$

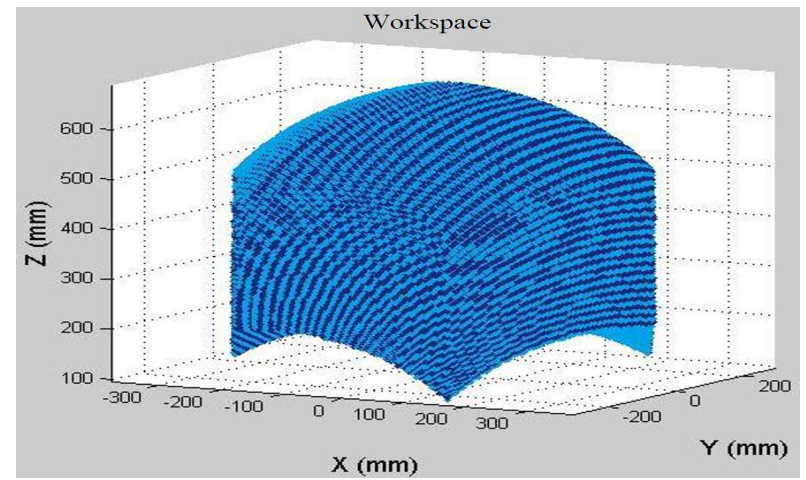

Figure 12: Workspace of the Delta Robot.

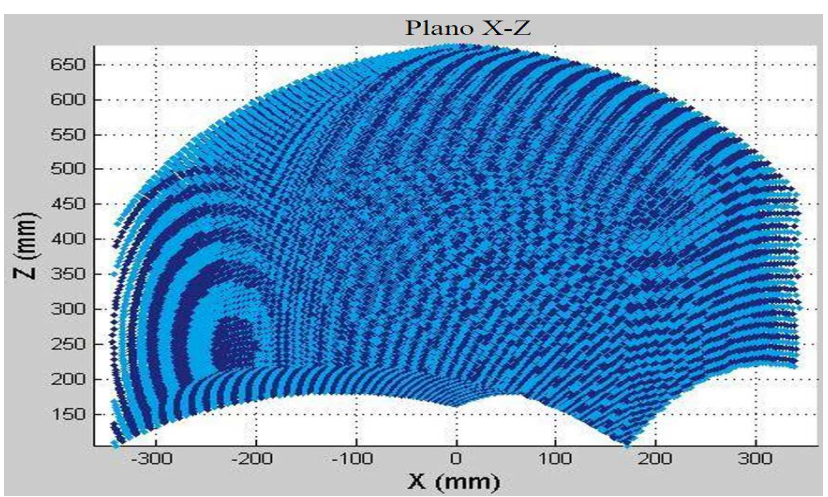

Figure 14: View of the Workspace of the Robot in $\mathrm{XZ}$ plane

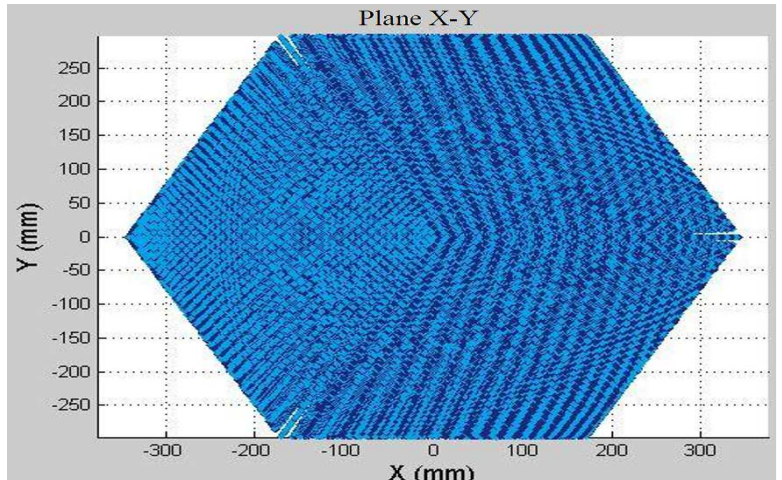

Figure 13: Top View of the Robot Workspace.

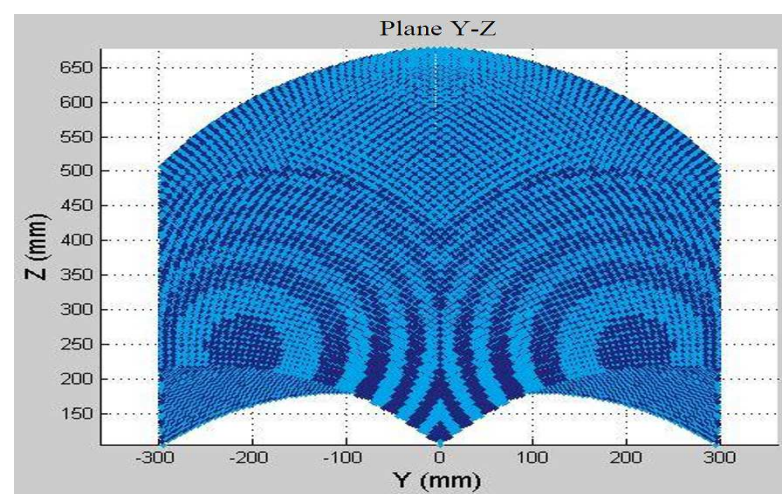

Figure 15: View of the Workspace of the Robot in YZ

\section{CONCLUSIONS}

The direct and inverse kinematics of a Delta robot can be solved by analyzing the geometry of the robot and posing equations that relate its dimensions, the angular position of the actuators and the position of the mobile base. Some of the most important characteristics to consider when designing a parallel robot are its workspace and its skill, given that these are the two shortcomings of these robots compared to serial manipulators.

As the workspace of parallel robots is composed of irregular volumes, it is necessary to rely on software, Matlab in this case, to be able to visualize all the points that the robot's mobile platform is able to reach. It was once again verified that genetic algorithms are very useful when solving optimization problems thanks to their robustness and easy implementation. In this case, not a single algorithm was implemented, but 18 algorithms to find the dimensions of the robot links that best show a balance between workspace and skill. The results obtained were dimensions of $260 \mathrm{~mm}$ and $420 \mathrm{~mm}$ 
with which the robot can reach almost 260000 points in the space of which 146273 points are optimal, that is, they comply with a local skill factor greater than 0.5 . Also, the overall skill of the robot is 0.556 , which meets the objective of designing a robot to maximize its workspace and its skill.

\section{ACKNOWLEDGEMENT}

Authors gratefully acknowledge the support of Lucas Urrea Mantilla, Sergio Alejandro Medina for their valuable support. Also, the research for paper was supported by Universidad Militar Nueva Granada, through the project ING-IMP-2657 ("Platform for the evaluation of therapeutic adherence and its influence on the efficiency of stabilometry therapies").

\section{REFERENCES}

1. S. B. Niku, Introduction to Robotics: Analysis, Control, Applications, 2 a ed. Wiley, 2010.

2. L.-W. Tsai, Robot Analysis: The Mechanics of Serial and Parallel Manipulators, la. ed. Wiley-Interscience, 1999.

3. M. Groover, M. Weiss, R. Nagel, y N. Odrey, Robótica industrial: tecnología, programación y aplicaciones. Madrid, España: McGrawHill, 1990.

4. D. Zhang, Parallel Robotic Machine Tools. Springer, 2009.

5. Y. Lou, G. Liu, J. Xu, y Z. Li, «A general approach for optimal kinematic design of parallel manipulators», presented at the 2004 IEEE International Conference on Robotics and Automation (ICRA'04), New Orleans, LA, 2004, vol. 4, pp. $3659-3664$.

6. L.-W. Tsai, G. C. Walsh, y R. E. Stamper, «Kinematics of a novel three DOF translational platform», presented at the 1996 IEEE International Conference on Robotics and Automation, Minneapolis, Minnesota, 1996, vol. 4, pp. 3446-3451.

7. J. Hong y M. Yamamoto, «A calculation method of the reaction force and moment for a Delta-type parallel link robot fixed with a frame», Robotica, vol. 27, no. 4, pp. 579-587, jul. 2009.

8. Z. Jingjun, S. Lihong, G. Ruizhen, y L. Chaoyang, «The mathematical model and direct kinematics solution analysis of Delta parallel robot», presented at the Second IEEE International Conference on Computer Science and Information Technology (ICCSIT), Beijing, China, 2009, pp. 450-454.

9. S.-D. Stan, M. Manic, V. Maties, y R. Balan, «Kinematics analysis, design, and control of an Isoglide3 Parallel Robot (IG3PR)», presented at the Thirtieth Annual Conference of IEEE Industrial Electronics (IECON 2008), Orlando, FL, 2008, pp. 2636-2641.

10. Xin-Jun Liu y Jongwon Kim, «A new three-degree-of-freedom parallel manipulator», presented at the 2002 IEEE International Conference on Robotics and Automation (ICRA'02), Washington, D.C., 2002, vol. 2, pp. 1155- 1160.

11. X.-J. Liu y J. Kim, «A new spatial three-DoF parallel manipulator with high rotational capability», IEEE/ASME Transactions on Mechatronics, vol. 10, no. 5, pp. 502- 512, oct. 2005.

12. M. A. Laribi, L. Romdhane, y S. Zeghloul, «Analysis and dimensional synthesis of the DELTA robot for a prescribed workspace», Mechanism and Machine Theory, vol. 42, no. 7, pp. 859-870, jul. 2007.

13. E. Martínez, C. Peña, y E. Yime, «Diseño óptimo de un robot paralelo con configuración Detla para aplicaciones educativas», Revista Educación en Ingeniería, no. 10, pp. 110-119, dic-2010.

14. M. Ceccarelli y C. Lanni, «A multi-objective optimum design of general $3 R$ manipulators for prescribed workspace limits», Mechanism and Machine Theory, vol. 39, no. 2, pp. 119-132, feb. 2004. 
15. L. Yunjiang, L. Guanfeng, C. Ni, y L. Zexiang, «Optimal design of parallel manipulators for maximum effective regular workspace», in 2005 IEEE/RSJ International Conference on Intelligent Robots and Systems (IROS 2005), 2005, pp. 795- 800.

16. L. A. Silva, J. M. Sebastian, R. Saltaren, R. Aracil, y J. Sanpedro, «RoboTenis: optimal design of a parallel robot with high performance», presented at the IEEE/RSJ International Conference on Intelligent Robots and Systems (IROS ), 2005, pp. 2134- 2139.

17. M. Badescu, J. Morman, y C. Mavroidis, «Workspace optimization of 3-UPU parallel platforms with joint constraints», in IEEE International Conference on Robotics and Automatio (ICRA '02), Washington, D.C., 2002, vol. 4, pp. $3678: 3683$.

18. R. E. Stamper, «A Three Degree of Freedom Parallel Manipulator with Only Translational Degrees of Freedom», University of Maryland, College Park, Maryland, 1997

19. Ramprasad Kannemadugu \& A. Lakshmi Devi, "Performance Improvement of Transmission System Using UPFC by GA and PSO Algorithms”, International Journal of Electrical and Electronics Engineering (IJEEE), Vol. 3, Issue 1, pp. 1-8

20. Ranu Paul, "Stability Analysis of Critical Points to Control Growth of Tumor in an Immune-Tumor-Normal Cell-Drug Model”, International Journal of Applied Mathematics \& Statistical Sciences (IJAMSS), Vol. 5, Issue 6, pp. 43-52

21. K. Rajeev \& K. Supraja, “A Study of Intelligent Controllers Application in Distributed Systems”, International Journal of Mechanical Engineering (IJME), Vol. 5, Issue 4, pp. 53-62

22. Amitvikram Nawalagatti \& Prakash R. Kolhe, "A Comparative Study on Artificial Cognition and Advances in Artificial Intelligence for Social-Human Robot Interaction”, International Journal of Robotics Research and Development (IJRRD), Vol. 8, Issue 1, pp. 1-10

\section{AUTHOR'S PROFILE}

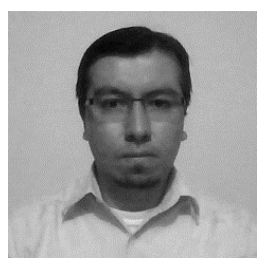

Ricardo A. Castillo E. was born in Colombia in 1980. He received his B.Eng. in Mechatronics Engineering in Universidad Militar Nueva Granada in 2004, and his M.Sc. and Ph.D. in Mechanical Engineering in the University of Campinas: UNICAMP, Brazil in 2010 and 2015 respectively, working on coordination and indirect communication strategies for mechatronic systems. Since 2005, he is a full-time Professor and Researcher in the Department of Mechatronics Engineering at Universidad Militar Nueva Granada (Bogotá: Colombia). His current research projects deal with collaborative modular robotics, artificial intelligence and mobile autonomous robotics.

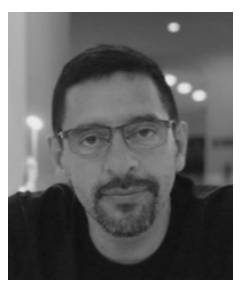

Oscar F Avilés S. He received the Engineer degree in Electronics and Specialist of Electronic Instrumentation of Antonio Nariño University: UAN - in 1995 and 2002, respectively. Master in Automatic Production Systems - Technological University of Pereira - 2006 Colombia and PhD in Mechanical Engineering in Campinas State University: UNICAMP: 
Brazil. Currently, he is Professor in the Department of Mechatronics Engineering in Universidad Militar Nueva Granada UMNG. He has experience in the areas of Electronic Engineering, with emphasis on electronic instrumentation and control systems, acting mainly on the following topics: robotics, control and biomechatronics.

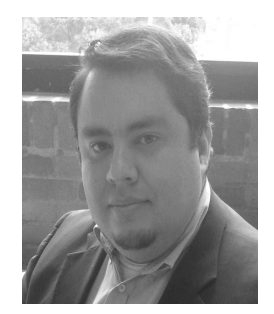

Mauricio Mauledoux He received the Engineer degree in Mechatronic engineering from the Universidad Militar Nueva Granada, in 2005. In 2008 he was a student of the Master in Information Technologies and Intelligent Systems in the St. Petersburg State Polytechnic University, Russia, at the automatic and intelligent distributed control department. He was then promoted to a $\mathrm{PhD}$. In 2011, he received the $\mathrm{PhD}$ degree in Mathematical models, numerical methods and software systems (Red Diploma) from the St. Petersburg State Polytechnic University, Russia. In 2012, he joined the Department of Mechatronic Engineering, at Universidad Militar Nueva Granada, in Colombia, as an Assistant Professor. His current research interests include Robotics, automatic control, Multi-agent Systems, Smart Grids, and Optimization. 